\title{
La hospitalización domiciliaria ante los cambios demográficos y nuevos retos de salud
}

\author{
Rosângela Minardi Mitre Cotta, ${ }^{1}$ María Morales Suárez-Varela, ${ }^{2}$ José Sette Cotta Filho, ${ }^{3}$ \\ Agustín Llopis González, ${ }^{2}$ José Antonio Días Ricós, ${ }^{4}$ y Enrique Ramón Real
}

RESUMEN Objetivos. En la actualidad, el fenómeno demográfico más importante es el rápido envejecimiento de la población, que tiene un creciente y profundo impacto en todos los ámbitos de la sociedad, aunque su mayor trascendencia es en la sanidad, tanto por su repercusión en todos los niveles asistenciales como por la necesidad de nuevos recursos y estructuras. De ahí que se hayan desarrollado en muchos países diferentes alternativas y programas institucionales, ambulatorios y domiciliarios, encaminados a mejorar la situación sanitaria y a auxiliar en el proceso de establecer prioridades. La hospitalización domiciliaria (HD) es una de ellas. El objetivo de este estudio consistió en describir y analizar las características de la población asistida en $H D$, y en comentar el papel de la HD como mecanismo de integración y coordinación entre niveles, frente al reto de la reorganización de políticas y proyectos de atención sanitaria, especialmente los dirigidos a la población anciana.

Métodos. Se realizó un estudio descriptivo, retrospectivo, de una serie de pacientes asistidos en HD en el área sanitaria número 9 de la Comunidad Valenciana, España, con una población de referencia de 321361 habitantes, de los cuales 60079 (18,7\%) son personas de 60 años o más, y 43044 (13,4\%), de 65 años o más. Se realizó un estudio descriptivo de las variables analizadas, calculándose la media y la desviación estándar para las variables cuantitativas, y las frecuencias absoluta y relativa (porcentaje) para las variables cualitativas.

Resultados. El perfil de los pacientes estudiados corresponde a ancianos (el $78 \%$ con 65 años o más; media de 73 años), predominantemente del sexo femenino, con enfermedades crónicas (72\%) y múltiples enfermedades asociadas (el 67\% tenía al menos un diagnóstico secundario asociado). Se constató la existencia de un importante problema de comunicación entre los dos principales niveles de atención (primaria y hospitalaria), que obviamente repercute en los pacientes y en la calidad y eficacia de la asistencia sanitaria. Asimismo, se verificó que la HD encuentra todo su significado en la población adulta o anciana con múltiples enfermedades crónicas degenerativas o terminales, en la que ha demostrado ser una herramienta eficiente. Conclusiones. Se destaca la necesidad de crear o potenciar los canales y mecanismos de comunicación interinstitucional que garanticen la continuidad del proceso asistencial. La asistencia continua y eficaz de la salud y bienestar del anciano requiere diferentes niveles de intervención sanitaria y debe estar basada en lo que hoy es un objetivo inaplazable: la atención integral, adecuada, de calidad, humanizada, oportuna y basada en una asistencia integrada y coordinada entre los dos principales niveles de atención sanitaria. Son estos factores los que, en última instancia, determinan la calidad de la asistencia y la capacidad resolutiva de los problemas asistenciales planteados en la atención al paciente geriátrico.

Palabras clave Hospitalización domiciliaria; paciente geriátrico; cambio demográfico; cambio epidemiológico; coordinación; integración.

1 Departamento de Nutrição e Saúde, Universidade Federal de Viçosa, Viçosa, Minas Gerais, Brasil. Actualmente en el Departamento de Medicina Preventiva y Salud Pública, Universidad de Valencia, Valencia, España. La correspondencia debe enviarse a Rosângela Minardi Mitre Cotta a la si- guiente dirección: Universidad de Valencia. Facultad de Farmacia. Departamento de Medicina Preventiva y Salud Pública. Unidad de Salud Pública, Higiene y Sanidad Ambiental. Av. Vicent Andrés Estellés, s/n. 46100 Burjasot, Valencia, España.

Correo electrónico: rosangelaminardi@hotmail.com
2 Departamento de Medicina Preventiva y Salud Pública, Universidad de Valencia, Valencia, España.

3 Departamento de Medicina, Universidad de Valencia, Valencia, España.

4 Unidad de Hospitalización a Domicilio, Hospital Universitario Dr. Peset, Valencia, España. 
En las últimas décadas ha habido un notable aumento del grupo de más de 65 años, en el que la esperanza de vida ha experimentado un gran incremento (1). En 1997, la población española mayor de 65 años representaba el 15,9\% del total, para el año 2010 será del 17,9\%, y en 2015 llegará al 20,3\% (2). En Estados Unidos, pese a que la tasa de mortalidad para el conjunto de la población es más alta que en Japón y algunos países europeos, la supervivencia de los mayores de 80 años es más elevada, debido a la notable calidad de la atención especializada que los ancianos reciben en ese país (3).

No cabe duda de que el cambio demográfico tiene un creciente y profundo impacto en todos los ámbitos de la sociedad, pero es en la sanidad donde tendrá mayor trascendencia, tanto por su repercusión en todos los niveles asistenciales como por la necesidad de aplicar nuevos recursos y estructuras (1). Este creciente envejecimiento de la población y el progresivo descenso de las tasas de fecundidad han generado en muchos países un cambio en el tipo de demanda de servicios médicos y sociales $(4,5)$. Además, hay una correlación directa entre los procesos de transición demográfica y epidemiológica. El envejecimiento aumenta el riesgo de adquirir enfermedades e incapacidades, y con ello la necesidad de implementar políticas para mantener condiciones de vida y de asistencia sociosanitaria adecuadas para las personas ancianas.

Una de las consecuencias del actual aumento de la longevidad es el predominio de las enfermedades crónicas y sus complicaciones (6-8). A medida que aumenta el número de ancianos y la esperanza de vida, se hacen más frecuentes las complicaciones por enfermedades crónicas degenerativas (secuelas de accidentes vasculares cerebrales y fracturas por caídas, limitaciones provocadas por enfermedades cardiovasculares y enfermedades pulmonares obstructivas crónicas, dependencia causada por enfermedades mentales como la demencia de Alzheimer, por la diabetes o por enfermedades terminales, etc). Estas enfermedades son la principal causa de muerte en los ancianos y también sus problemas de salud más prevalentes, incrementando vertiginosamente la demanda asistencial y los costes sanitarios (9-12).

En ese contexto se plantea la necesidad de transformar y reorganizar los sistemas y servicios de salud, garantizando el acceso oportuno, adecuado y de calidad a toda la población. De ahí que se hayan desarrollado en muchos países diferentes programas y alternativas institucionales, ambulatorias y domiciliarias, encaminadas a mejorar la situación sanitaria y auxiliar en el proceso de establecer prioridades (13). La hospitalización domiciliaria (HD) es una de ellas.

La HD como modalidad asistencial no es nueva. Su inicio se remonta al año 1947, cuando se creó en los Estados Unidos la primera Unidad de "home care"; en 1951 surgió la primera experiencia en Europa (París), y a España llegó en 1982. Desde entonces, la HD viene cobrando cada vez más importancia en las distintas organizaciones sanitarias y sociales y en diferentes sectores de la sociedad (5). En los últimos años se ha despertado un gran interés por esta modalidad de prestación de servicios $(8,11)$, que se considera una alternativa asistencial halagadora, viable y prometedora desde el punto de vista sanitario, social y económico, y se preve un aumento creciente de la demanda de estos servicios $(12,14)$.

Este estudio tiene un doble objetivo: por un lado, describir y analizar las características clínicas de la población asistida en HD, así como la competencia y la estructura del servicio, $y$, por otro, analizar el papel de la HD frente al reto de reorganizar las políticas y los proyectos de asistencia sanitaria para la población anciana.

\section{MATERIALES Y MÉTODOS}

Se realizó un estudio descriptivo retrospectivo $(14,15)$ de una serie de pacientes asistidos en HD en el área sanitaria número 9 de la Comunidad Valenciana, España, entre abril y diciembre de 1999, período durante el cual se produjeron 799 ingresos en esta modalidad asistencial. La población de referencia del área sanitaria en estudio es de 321361 habitantes, de los cuales $60079(18,7 \%)$ son personas con 60 años o más, y $43044(13,4 \%)$ con 65 años o más. La figura 1 muestra la pirámide de población del área (16-18).

De acuerdo con la literatura y dadas las características demográficas y epidemiológicas de los pacientes de nuestro estudio, estos se han clasificado en tres grupos patológicos: "procesos agudos", "procesos crónicos" y "otros/ cuidados paliativos". En el grupo con "procesos agudos" destacan como principales afecciones las neumonías, pielonefritis, aplasia posquimioterapia $y$ aquellas enfermedades que pueden ser tratadas con antibióticos intravenosos en el domicilio (19-23). En el grupo con "procesos crónicos" destacan las enfermedades pulmonares obstructivas crónicas, cardiopatías, hepatopatías crónicas, síndrome de inmunodeficiencia adquirida, enfermedades terminales, diabetes y secuelas de accidentes vasculares cerebrales $(20,22$, 24). En la categoría "otros/cuidados paliativos" se incluyen aquellos pacientes que necesitan de una estructura de tecnología y farmacia hospitalaria, como pruebas diagnósticas especiales, interconsultas, etc., y se considera que un paciente requiere cuidados paliativos cuando está afectado por una enfermedad terminal, en fase avanzada, progresiva e incurable, con presencia de numerosos problemas y síntomas y un pronóstico de vida igual o inferior a 6 meses $(19,21-23)$.

De acuerdo con los objetivos fijados, las variables analizadas se dividieron en dos categorías: variables clínicas y variables relacionadas con la estructura y competencia del servicio. Con respecto a la estructura etaria, solo se analizaron los datos de 790 pacientes; en el 1,1\% restante los datos no estaban claros.

Se realizó un estudio descriptivo de las variables analizadas; se calcularon las medias y las desviaciones estándar (DE) de las variables cuantitativas y las frecuencias absolutas y relativas (porcentajes) de las variables cualitativas. Las proporciones se compararon con la prueba de la $x^{2}$; se consideraron significativas las diferencias con 
FIGURA 1. Pirámide de población del Área Sanitaria $n^{\circ} 9$ de la Comunidad Valenciana, España, 1999

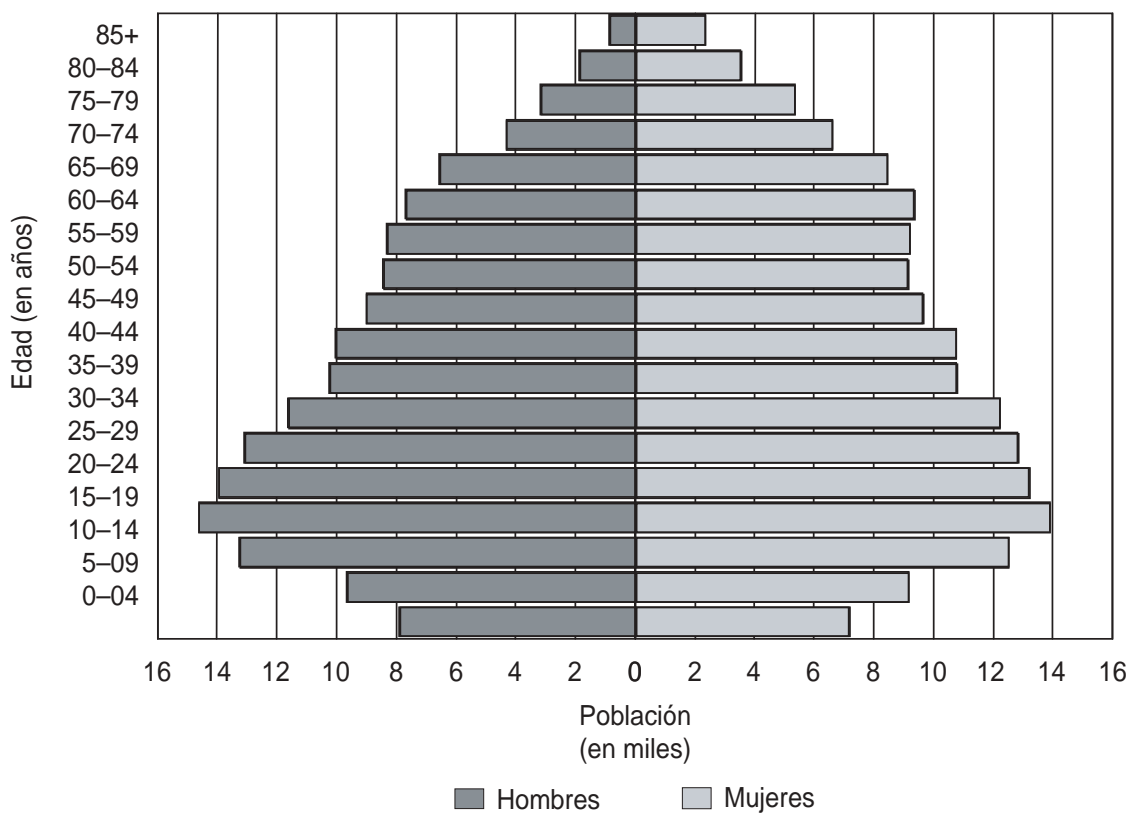

Población Total $=321361$ (hombres $=154825$ y mujeres $=166$ 536)

Fuente: Memòria. Hospital Universitari Dr. Peset-Centre d’especialitats Monteolivete.

un valor $P<0,05$. Para el análisis estadístico se utilizó el programa informático SPSS, versión 8.0 (Chicago, IL, Estados Unidos).

\section{RESULTADOS}

\section{Características de los sujetos y variables clínicas}

La figura 2 muestra la distribución de los sujetos asistidos por edad y sexo. Hubo un discreto predominio del sexo femenino: 401 mujeres $(50,7 \%)$, frente a $389(49,3 \%)$ hombres $(P=0,0486)$. La edad de los pacientes osciló entre 19 y 100 años, con una media $( \pm \mathrm{DE})$ de $73 \pm$ 14,6 años y una mediana de 76,0 años. La media fue de 75,3 $\pm 13,4$ años para las mujeres y de 70,2 $\pm 15,3$ años para los hombres. El 22\% de los pacientes eran menores de 65 años y el 78\% tenían 65 años o más. De estos últimos, el $16 \%$ eran mayores de 85 años. Entre los menores de 65 años predominaron los hombres, pero la situación se invirtió a medida que aumentó la edad, obser- vándose un aumento gradual del por centaje de mujeres, lo cual puede justificarse por su mayor supervivencia en comparación con los hombres.
La figura 3 muestra la distribución de frecuencias de los tres grupos patológicos según el sexo. Un $28 \%$ de los pacientes $(n=225)$, con una media de $76 \pm 13,6$ años, ingresaron por "procesos agudos" y un 47\% $(n=367)$, con una media de $73 \pm 11,5$ años, por "procesos crónicos". El 25\% restante ( $n=198)$, con una media de $67 \pm 19,1$ años, se incluyó en la categoría de "otros/cuidados paliativos".

Además del diagnóstico principal, 526 de los pacientes ingresados en HD (67\%) presentaron por lo menos un diagnóstico secundario: neoplasias en un $27,0 \%$, enfermedades del aparato circulatorio en un $14,6 \%$, enfermedades endocrinas en un $9,9 \%$, trastornos mentales en un 9,5\%, enfermedades del aparato genitourinario en un 3,5\% y otros grupos patológicos no especificados en un $35,4 \%$.

\section{Variables relacionadas con la competencia y estructura del programa}

El cuadro 1 muestra la procedencia de los pacientes ingresados en HD según los grupos patológicos. Más de la mitad (53\%) de los pacientes procedían de la unidad médica de estancias
FIGURA 2. Distribución de los pacientes atendidos en hospitalización domiciliaria, según edad y sexo. Comunidad Valenciana, España, 1999

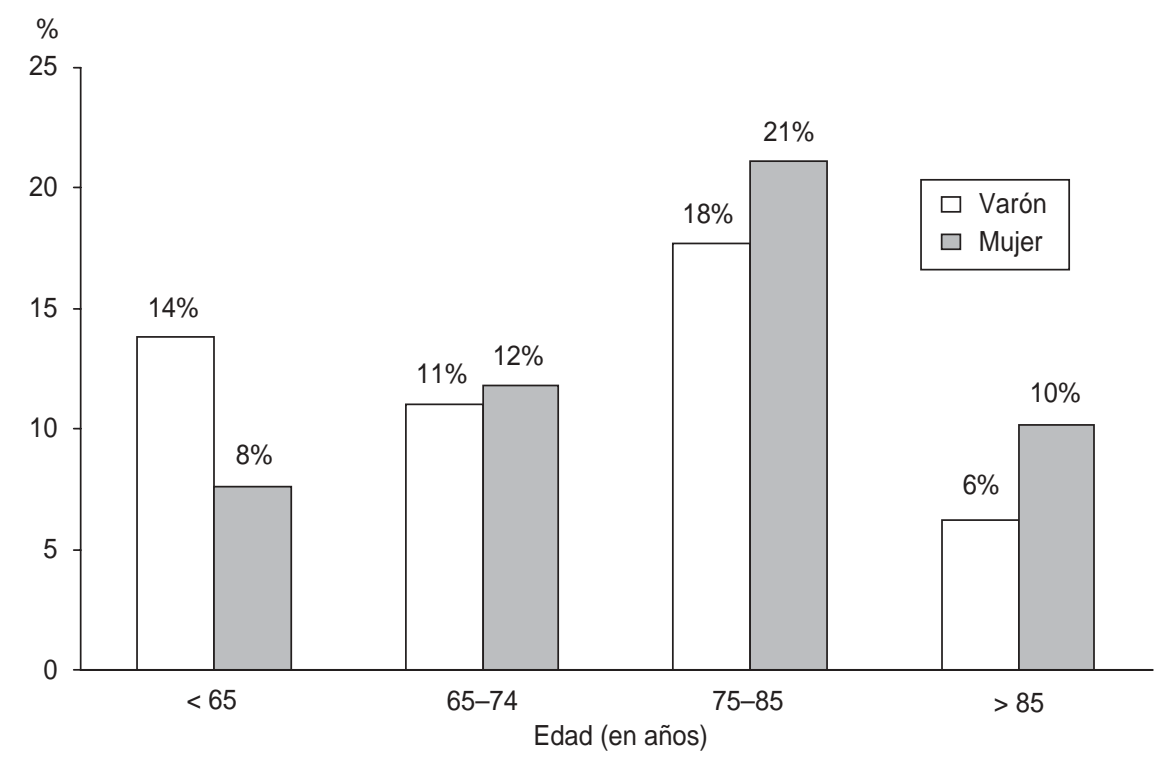


FIGURA 3. Distribución de frecuencias según grupos patológicos y sexo. Comunidad Valenciana, España, 1999

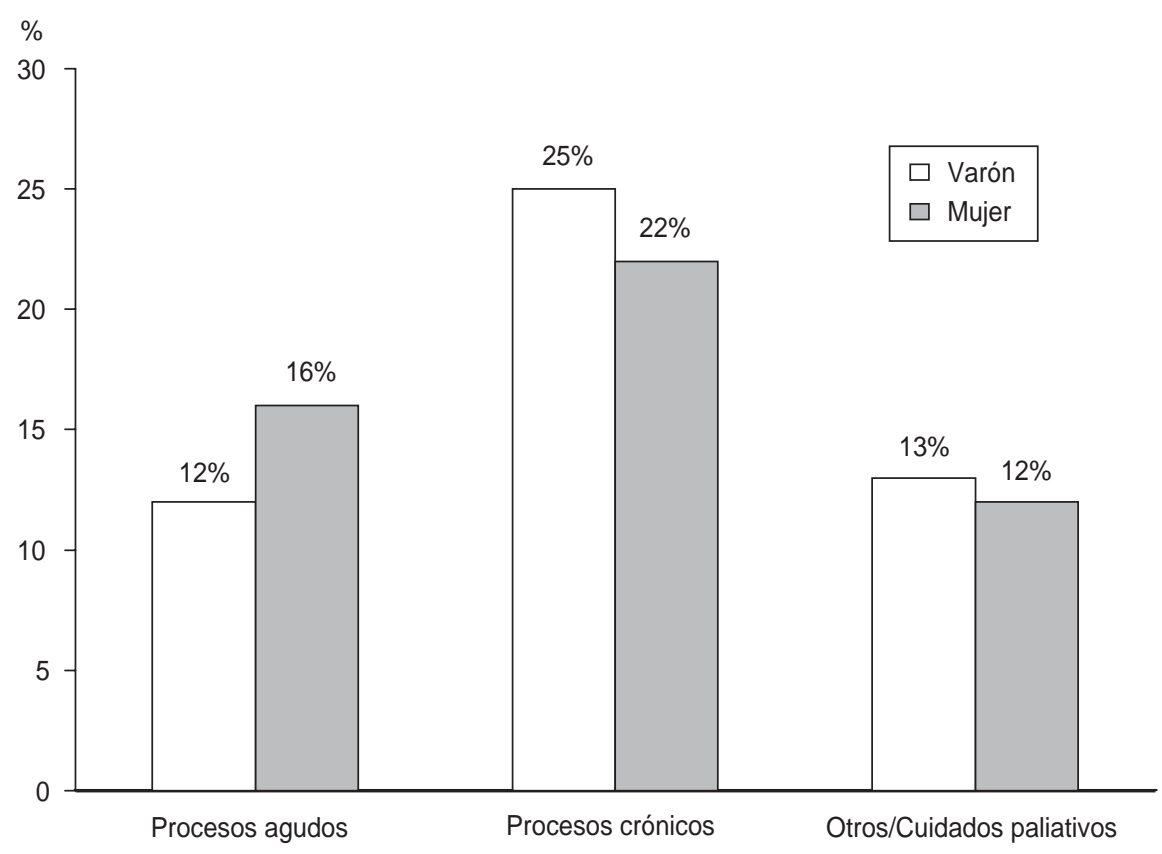

cortas (UMEC) y un 20\% del hospital, después de recibir el alta de la hospitalización tradicional.

El cuadro 2 ilustra el destino de los pacientes tras recibir el alta de la HD, según los grupos patológicos. En su mayoría fueron derivados a la atención primaria $(62 \%)$.
En general, la estancia media en HD fue de $8 \pm 11,2$ días: $8,3 \pm 8,1$ días para los ingresados con "procesos agudos", $8,3 \pm 14,2$ días para los ingresados con "procesos crónicos" y 7,3 $\pm 7,1$ días para los pacientes cuya causa de ingreso se encuadra en la categoría de "otros/cuidados paliativos".

CUADRO 1. Procedencia de los pacientes ingresados en hospitalización domiciliaria según los grupos patológicos. Comunidad Valenciana, España, 1999

\begin{tabular}{|c|c|c|c|c|c|c|c|c|c|}
\hline \multirow[b]{2}{*}{ Procedencia } & \multirow{2}{*}{$\begin{array}{c}\text { Edad } \\
\text { (media } \pm \mathrm{DE})\end{array}$} & \multicolumn{2}{|c|}{$\begin{array}{c}\text { Procesos } \\
\text { agudos }\end{array}$} & \multicolumn{2}{|c|}{$\begin{array}{l}\text { Procesos } \\
\text { crónicos }\end{array}$} & \multicolumn{2}{|c|}{$\begin{array}{l}\text { Otros/cuidados } \\
\text { paliativos }\end{array}$} & \multicolumn{2}{|c|}{ Total } \\
\hline & & $n$ & $\%$ & $n$ & $\%$ & $n$ & $\%$ & $n$ & $\%$ \\
\hline UMECa & $76 \pm 11,4$ & 181 & 23,0 & 194 & 24,0 & 49 & 6,0 & 423 & 53 \\
\hline Hospital $^{b}$ & $71 \pm 14,9$ & 19 & 2,3 & 81 & 10,0 & 63 & 7,7 & 163 & 20 \\
\hline Autoderivación & $79 \pm 11,8$ & 7 & 0,9 & 25 & 3,3 & 14 & 1,8 & 46 & 6 \\
\hline Atención primaria & $76 \pm 12,8$ & 6 & 1,0 & 16 & 2,0 & 14 & 2,0 & 36 & 5 \\
\hline Hospital de día & $68 \pm 9,3$ & - & - & 25 & 3,0 & 8 & 1,0 & 33 & 4 \\
\hline Urgencias & $75 \pm 10,5$ & 8 & 0,8 & 7 & 0,7 & 5 & 0,5 & 20 & 2 \\
\hline Otros $^{c}$ & $53 \pm 19,2$ & 4 & 0,5 & 21 & 2,7 & 53 & 6,8 & 78 & 10 \\
\hline Total & $73 \pm 14,6$ & 225 & 28,5 & 351 & 43,7 & 223 & 27,8 & 799 & 100 \\
\hline
\end{tabular}

a Unidad médica de estancias cortas.

b Tras recibir el alta de la hospitalización tradicional.

${ }^{c}$ Residencias asistidas, consultas externas, otros hospitales, servicio social.

DE: desviación estándar.

\section{DISCUSIÓN}

El perfil de los pacientes estudiados corresponde a ancianos ingresados por procesos crónicos, con múltiples enfermedades asociadas y predominio del sexo femenino, cuyo porcentaje va aumentando con la edad (figuras 2 y $3)$. Estos datos son similares a los obtenidos en otros estudios $(10,25-28)$ que prevén un incremento de los programas de atención domiciliaria en proporción con el envejecimiento de la población y concluyen que la atención sociosanitaria a domicilio es el espacio capaz de ofrecer una asistencia global e integrada, en especial a los ancianos, optimizando los recursos y generando sinergias tanto entre la atención primaria y el hospital como entre la atención sanitaria y social, potenciando y facilitando así una acción más coordinada integrada entre los diferentes niveles sociosanitarios $(4,29)$.

En la misma línea, otros autores (2933) resaltan que los pacientes de edad avanzada, portadores de síndromes geriátricos $\mathrm{u}$ otros problemas clínicos que, por su complejidad y gravedad, son importantes factores de riesgo de ingreso hospitalario, pueden superar las posibilidades de tratamiento en el ámbito de la atención primaria y son especialmente indicados para ser atendidos en HD. Asimismo, la presencia de múltiples enfermedades concomitantes es un hallazgo constante en los estudios sobre la $\operatorname{HD}(23,34,35)$.

Los ancianos representan una fracción muy importante de la población en el área geográfica estudiada (figura 1), lo cual constituye un factor sociodemográfico fundamental que influye en la asignación de los recursos sociosanitarios, como señalan gran parte de los trabajos publicados (1, 9, 36-39) que abordan el fenómeno del envejecimiento de la población y sus importantes consecuencias sobre el sistema de salud. Asimismo, otros autores han señalado que, por su importante morbilidad (predominio de enfermedades crónicas degenerativas y sus complicaciones), los ancianos corren un alto riesgo de ingreso hospitalario y consumen 5 a 10 veces más recursos sanita- 
CUADRO 2. Destino de los pacientes tras el alta de hospitalización domiciliaria según los grupos patológicos. Comunidad Valenciana, España, 1999

\begin{tabular}{|c|c|c|c|c|c|c|c|c|c|}
\hline \multirow[b]{2}{*}{ Procedencia } & \multirow{2}{*}{$\begin{array}{c}\text { Edad } \\
\text { (media } \pm \mathrm{DE})\end{array}$} & \multicolumn{2}{|c|}{$\begin{array}{l}\text { Procesos } \\
\text { agudos }\end{array}$} & \multicolumn{2}{|c|}{$\begin{array}{l}\text { Procesos } \\
\text { crónicos }\end{array}$} & \multicolumn{2}{|c|}{$\begin{array}{l}\text { Otros/cuidados } \\
\text { paliativos }\end{array}$} & \multicolumn{2}{|c|}{ Total } \\
\hline & & $n$ & $\%$ & $n$ & $\%$ & $n$ & $\%$ & $n$ & $\%$ \\
\hline Atención primaria & $75 \pm 12,4$ & 185 & 23,0 & 245 & 31,0 & 63 & 8,0 & 492 & 62 \\
\hline Muerte & $75 \pm 12,3$ & 5 & 0,6 & 22 & 2,8 & 76 & 9,6 & 103 & 13 \\
\hline \multicolumn{10}{|l|}{ Reingreso en } \\
\hline el hospital & $73 \pm 10,5$ & 17 & 2,0 & 47 & 5,7 & 11 & 1,3 & 75 & 9 \\
\hline Consultas externas & $65 \pm 16,5$ & 13 & 1,7 & 26 & 3,3 & 8 & 1,0 & 47 & 6 \\
\hline Otros $^{a}$ & $48 \pm 17,6$ & 7 & 0,9 & 35 & 4,3 & 40 & 4,8 & 82 & 10 \\
\hline Total & $73 \pm 14,6$ & 225 & 28,5 & 351 & 43,7 & 223 & 27,8 & 799 & 100 \\
\hline
\end{tabular}

a Residencia asistidas, consultas externas, otros hospitales, servicio social.

DE: desviación estándar.

rios que los de menor edad $(5,9,20,23$, 27, 36).

En ese sentido, la elevada media de edad de los pacientes de este estudio hace pensar que la HD no trabaja con toda la población susceptible de utilizar este recurso. Es decir, al priorizarse los pacientes ancianos se excluyen los más jóvenes $(6,16,24,40)$.

Una posible limitación de nuestro estudio proviene del hecho de que no hemos identificado la causa de esa preselección de los pacientes derivados a $\mathrm{HD}$, lo cual puede deberse a varios factores, como una decisión previa de especialización del equipo de $\mathrm{HD}$, una demanda basada en un estudio previo del perfil epidemiológico y demográfico del área sanitaria, una sobrecarga del equipo de HD, o quizás una utilización sesgada de la HD por el resto de los servicios sanitarios, principalmente los hospitalarios, que son los que más pacientes derivan y que podrían estar enviando solo a determinados grupos de pacientes, los más "pesados o incómodos", desaprovechando así el potencial de los equipos de HD $(28,41)$.

Desde esta perspectiva, el establecimiento del "mapa de riesgo" 5 podría

\footnotetext{
5 El mapa de riesgo se define como la distribución geográfica de los individuos, familias o comunidades en riesgo. Las unidades de estudio son las diferentes demarcaciones de la "zona básica de salud" según sus características socioeconómicas y culturales (42).
}

constituir un instrumento útil para identificar la distribución espacial de los problemas de salud y de la necesidad de servicios que de ella se deriva, permitiendo en nuestro caso conocer los factores de riesgo de la población anciana o portadora de enfermedades crónicas degenerativas (42). Esta modalidad de "mapa de riesgo" puede ser el punto de partida para el establecimiento de programas de atención domiciliaria coordinados e integrados entre los equipos de los dos niveles principales de atención sanitaria: el hospital y la atención primaria.

En todo caso, hay que destacar que en la mayoría de los países donde se ha implantado esta modalidad asistencial, la HD se dedica básicamente a la asistencia a los ancianos o a pacientes con cáncer o enfermedades degenerativas que evolucionan a la muerte, $\mathrm{y}$ a los cuidados paliativos $(23,37,43,44)$. Quizás esto sea así, en parte, debido a que estos tipos de pacientes cumplen todas las características que debe tener la HD (transitoriedad, intensidad y complejidad de la asistencia) (45-48), y más especialmente la característica de la complejidad del proceso terapéutico o patológico y de la utilización de técnicas diagnósticas y terapéuticas que deben ser propias del hospital y realizadas por personal especializado.

Con respecto a los procesos patológicos, las enfermedades crónicas son la principal causa de ingreso en HD $(47 \%$ del total), lo cual se explica por el per- fil etario de los pacientes. De hecho, este porcentaje es mucho mayor, ya que habría que añadirle el $25 \%$ de la categoría "otros/cuidados paliativos". Es decir, en este estudio, el $72 \%$ de los enfermos presentaban enfermedades crónicas (figura 3).

El análisis de la procedencia y destino de los pacientes ingresados en HD aporta datos interesantes para la reflexión sobre el polémico tema de la integración y coordinación entre la atención primaria y hospitalaria y la continuidad de la asistencia.

Con respecto a la procedencia (cuadro 1), se observa que la comunidad tiene una participación poco significativa en la elección de la HD como alternativa asistencial. El $79 \%$ procede de servicios ligados a la atención especializada $\mathrm{y}$, más específicamente, al hospital (UMEC, hospitalización tradicional, hospital de día o urgencias). En realidad, este porcentaje puede ser todavía mayor, ya que en la categoría "otros", que representa un $10 \%$, algunos proceden de la atención especializada, como pueden ser las consultas externas y otros hospitales. Estos datos indican que la atención primaria tiene una participación poco significativa en la derivación de los pacientes hacia la HD y, por lo tanto, nos encontramos ante un sistema que se ha organizado desde el propio hospital para enviar a los pacientes al servicio de HD.

En la literatura científica revisada no son pocos los estudios que resaltan el hecho de que, hoy por hoy, el sistema sanitario español gira aún en torno a los hospitales $(6,7,11,23,24)$, siendo España uno de los países europeos más orientados hacia la atención especializada y, más concretamente, la hospitalaria $(7,28,43,49)$. Ese fenómeno se refleja tanto en las cifras presupuestarias (el $76 \%$ del gasto sanitario está concentrado en los hospitales) como en el diseño de los incentivos a los profesionales hospitalarios y en su prestigio y reconocimiento por parte del sector y de los ciudadanos en general (50-52).

Sin embargo, hay que destacar que, con matices locales, el problema del excesivo protagonismo del hospital en 
detrimento de los servicios de atención primaria es extensible a casi todos los países y, desde luego, no está resuelto satisfactoriamente en ninguna parte $(28,40,53)$. También hay coincidencia en el diagnóstico de las deficiencias en los resultados de los servicios de atención primaria, destacando sobre todo su incapacidad para reducir las urgencias hospitalarias y su limitada capacidad de contener el acceso a la atención hospitalaria $(7,11,28,42)$. El excesivo protagonismo del hospital y, por consiguiente, la menor importancia relativa de la atención primaria tienen como resultado el perjuicio de la calidad resolutiva de la asistencia en general (clínica, organizativa y financiera).

Por otro lado, el análisis del destino de los pacientes tras la HD (cuadro 2) revela que un porcentaje importante de ellos $(62 \%)$ son encaminados hacia la atención primaria para continuar el tratamiento y que los demás son derivados a los servicios hospitalarios o mueren. Considerando el perfil etario y epidemiológico de la población estudiada (ancianos con una media de 73 años, en su mayoría portadores de enfermedades crónicas degenerativas e invalidantes), el análisis del destino de los pacientes y de los días de estancia en HD, cuya media de 8 días es inferior a los 10,6 días de estancia media de los mayores de 65 años en la hospitalización tradicional (42), parece sugerir una buena calidad de la asistencia prestada en la $\operatorname{HD}(9,31,35)$. Es decir, al cumplir con la característica de transitoriedad (45-48) y al encaminar una parte considerable de los pacientes hacia los servicios de atención primaria, la HD parece ser un recurso eficaz y oportuno como alternativa a la hospitalización tradicional.

Parece lógico pensar que hay un problema de comunicación entre los dos principales niveles sanitarios, que obviamente repercute en los pacientes y en la calidad y eficacia de la asistencia sanitaria prestada $(7,23,28,53)$. En todo caso, el problema de la insuficiente coordinación e integración entre la atención primaria y la atención especializada no es una característica únicamente del sistema sanitario español, ni tampoco de la HD, sino más bien una de las críticas que tradicionalmente se hacen en casi todos los países que implementaron reformas sanitarias, destacándose como un de los principales retos sociosanitarios pendientes $(28,50-53)$. De hecho, pese a que en todos estos países la aplicación paulatina de la reforma sanitaria prevé un fortalecimiento progresivo de los servicios y equipos de atención primaria, que debería ser la puerta de entrada al sistema, y el consiguiente establecimiento de alianzas estratégicas entre la atención primaria y el hospital en favor de un sistema planificado, coordinado e integrado de salud, en la práctica estamos muy lejos de ese esquema idílico $(28,30,31,48)$.

Hemos de reconocer, sin embargo, el papel primordial que tiene la atención domiciliaria en la potenciación de la coordinación e integración de los servicios sanitarios y sociales, ya que permite compartir responsabilidades clínicas, organizativas y financieras, y superar la tradicional provisión dicotómica de la asistencia, con lo que puede constituirse en un elemento facilitador e impulsor de una progresiva conexión entre el hospital y la atención primaria $(28,42,46,54)$.

En ese contexto, el estudio desarrollado aquí pone de relieve la siempre difícil relación entre la atención primaria y la atención especializada. Cabe mencionar la discusión sobre la competencia de la atención domiciliaria (atención primaria $\mathrm{u}$ hospitalaria) planteada en diferentes estudios $(12,28,39,46)$. El hecho de que la HD desarrolle su labor en un sitio que tradicionalmente pertenece a la atención primaria (el domicilio del paciente), antes que ser motivo de discordia y rivalidad, debería favorecer la cooperación y la convergencia de intereses entre los equipos del hospital y de la atención primaria, ya que la HD debe cumplir unas características propias que la diferencian de la atención domiciliaria prestada por el equipo de atención primaria.

No cabe duda de que para determinados tipos de pacientes, como los de nuestro estudio, la HD encuentra todo su significado y ha demostrado ser una herramienta eficiente $(7,17,18$, 47). Muchos autores $(26,33,35,45,54)$ han destacado que, debido al envejecimiento creciente de la población y al consiguiente aumento de la demanda asistencial y sanitaria, la HD tiene grandes perspectivas de futuro y será una opción cada vez más utilizada, ya que permite que el anciano permanezca en su domicilio con la mejor calidad de vida posible.

No obstante, el desarrollo de la HD no debería llevarse a cabo de forma ajena a la atención primaria, constituyendo una red paralela de servicios. El éxito o el fracaso de la asistencia domiciliaria a pacientes con un perfil similar a los de nuestro estudio supondrá una inevitable reorganización de los servicios asistenciales, el cambio en la orientación de la formación de los profesionales, el diseño conjunto de protocolos o guías de actuación (preventiva, diagnóstica, terapéutica y de seguimiento y control), y el mejoramiento de la capacidad de coordinación e integración con la atención primaria, que debe ser fluida y disponer de cauces de comunicación y cooperación formales y bien definidos (53, 55-57).

Una asistencia continua y eficaz a la salud y bienestar del anciano requiere diferentes niveles de intervención sanitaria adecuados a las distintas fases de la enfermedad y al grado de incapacidad, y debe estar basada en lo que hoy es un objetivo inaplazable: la atención integral, adecuada, de calidad, humanizada y oportuna. La idea clave es converger hacía la superación de la impermeabilidad administrativa entre las diferentes instituciones sanitarias, buscando la proximidad entre la gestión y la provisión de servicios, potenciando la acción local y reconociendo y apoyando la provisión de asistencia domiciliaria, cuya relevancia está en alza en un contexto de progresivo envejecimiento de la población $(52,55$, 57). De hecho, estos factores son los que en última instancia determinan la calidad de la asistencia y la capacidad resolutiva de los problemas asistenciales planteados en la atención al paciente geriátrico $(51,52,56)$.

El presente trabajo ha puesto de manifiesto la importancia de la HD como alternativa asistencial, especialmente en contextos y áreas sanitarias donde el 
progresivo envejecimiento de la población es un factor de primordial relevancia demográfica y epidemiológica.

La discusión del tema adquiere especial importancia si consideramos que casi todos los países del continente americano y de la Unión Europea están reformando sus sistemas de salud, teniendo como factor clave la implementación de estrategias que propicien la transformación de los servicios para resolver con eficacia las inequidades de acceso y garantizar un acceso oportuno, adecuado y de calidad a toda la población.

En este trabajo se ha discutido también el papel que puede tener la HD en relación con las principales prioridades organizativas que afectan a los sistemas de salud y que se refieren a aspectos tales como la coordinación e integración entre atención primaria y especializada y entre atención sanitaria y sociosanitaria, la continuidad de la asistencia, la homogeneización y mejora de los sistemas de información y comunicación entre los diferentes niveles y la implicación de los pro- fesionales sanitarios en las reformas organizativas.

Aunque en nuestro estudio se ha comprobado la falta de integración entre la atención primaria y hospitalaria, concluimos que, establecidos los supuestos necesarios, como la elaboración de planes de actuación asistencial conjunta, el establecimiento de un marco administrativo adecuado para la atención y seguimiento de los pacientes, el diseño conjunto de protocolos o guías de actuación, la informatización de historias clínicas y la institucionalización de los profesionales de enlace responsables de la comunicación entre el hospital y la atención primaria, la HD puede llegar a ser un elemento de cohesión y contribuir a la superación de la histórica dicotomía, desintegración y descoordinación del sistema sanitario.

Hechas estas consideraciones, se puede concluir que la HD se justifica en función de la población a la que se destina y de sus necesidades sanitarias. Dado el escenario privilegiado en el que se desarrolla (domicilio y comu- nidad), tiene como ventaja añadida la apertura del hospital y el acercamiento de los profesionales y de las estructuras hospitalarias a la comunidad. Si logra encontrar puntos de colaboración y coordinación, puede llegar a potenciar y viabilizar un nuevo y necesario tipo de alianza y coalición en la política de salud del municipio o área sanitaria. Para esto, es necesario el compromiso y la responsabilización de todos los actores sociales involucrados en el proceso, es decir, los gestores locales, los médicos, los enfermeros y otros profesionales sanitarios y sociales del hospital y de la atención primaria, la familia y el paciente.

Agradecimientos. El contenido de este artículo se basa en investigaciones que fueron financiadas por el Conselho de Desenvolvimento Científico e Tecnológico (CNPq)/Brasil, proyecto no. 201178/97-4, y forma parte de un esfuerzo conjunto entre equipos de investigadores de Brasil y España dirigido a explorar gestiones innovadoras en sistemas sanitarios.

\section{REFERENCIAS}

1. Vaqué J. El aumento de la longevidad y su relación con la morbilidad. Med Prev 1998;4: 19-26.

2. EUROSTAT. Statistiques démographiques 1995. Luxembourg: Office des Publications Officelles des Communautés Européennes; 1995.

3. Manton KG, Vaupel JW. Survival after the age of 80 in the United States, Sweden, France, England, and Japan. N Engl J Med 1995;333: 1232-1235.

4. Jones J, Wilson A, Parker H, Wynn A, Jagger C, Spiers N, et al. Economic evaluation of hospital at home versus hospital care: cost minimisation analysis of data from randomised controlled trial. BMJ 1999;319:1547-1550.

5. Murray CJ, López AD. Regional patterns of disability-free life expectancy and disabilityadjusted life expectancy: global burden of disease study. Lancet 1997;349:1347-1352.

6. Saltman RB, Figueras J. European health care reform. Analysis of current strategies. Copenhagen: World Health Organization Regional Office for Europe; 1997.

7. Contel SJC. La atención a domicilio como modelo de atención compartida [Editorial]. Atención Primaria 2000;25:22-28.
8. Vita AJ, Terry RB, Hubert HB, Fries JF. Aging, health risks, and cumulative disability. $\mathrm{N}$ Engl J Med 1998;338:1035-1041.

9. Chaimowicz F. A saúde dos idosos brasileiros às vésperas do século XXI: problemas, projeções e alternativas. Rev Saude Publica 1997; 31:184-200.

10. Butler RN. Care of the aged in the United States of America. En: Tallis RC, Fillit SHM, Brocklehurst TJC (eds.). Textbook of geriatric medicine and gerontology (4th ed.). Edinburgh: Churchill Livingstone, 1993. pp. 993-999.

11. Contel SJC. La hospitalización a domicilio no es una alternativa plenamente razonable. Atención Primaria 1999;24:162-168.

12. Stewart S, Marley JE, Horowitz JD. Effects of a multidisciplinary, home-based intervention on unplanned readmissions and survival among patients with chronic congestive heart failure: a randomised controlled study. Lancet 1999;354:1077-1083.

13. Beyrer K, Brauer GW, Fliedner TM, Greiner C, Reischl U. Light on population health status. Bull World Health Organ 1999;77:176-180.

14. Argimón Pallas JM, Jiménez Villa J. Métodos de investigación aplicados a la atención pri- maria de salud. Barcelona: Mosby/Doyma Libros; 1995.

15. Hulley SB, Cummings SR. Diseño de la investigación clínica. Un enfoque epidemiológico. Madrid: Harcourt Brace Publisher International; 1997.

16. Hospital Universitari "Dr. Peset"-Centre d'especialitats "Monteolivete". Valencia. Memòria any 1997. [Sitio en Internet]. Disponible en: http://www.san.gva.es/memorias.htm (Acceso el 10 de abril de 2002).

17. Área de Salud No. 9. Atención primaria.Valencia. Memòria any 1996. [Sitio en Internet]. Disponible en: http://www.san.gva.es/memorias. htm (Acceso el 10 de abril de 2002).

18. Institut Valencià D’Estadística. Inebase 2001. [Sitio en Internet]. Disponible en: http://www. ine.es/daco/daco42/demograf

19. Davies TM. Future challenges for the general practitioner: hospital at home. En: The Royal College of General Practitioners Members' Reference Book. London: Campden Publishing, Ltd.; 1997.

20. Fried TR, van Doorn C, O'Leary JR, Tinetti $\mathrm{ME}$, Drickamer MA. Older person's preferences for home vs. hospital care in the treat- 
ment of acute illness. Arch Intern Med 2000; 160:1501-1506.

21. Jordhoy MS, Fayers P, Saltnes T, AhlnerElmqvist M, Jannert M, Kaasa S. A palliativecare intervention and death at home: a cluster randomised trial. Lancet 2000;356:888-893.

22. Hadley J, Rabin D, Epstein A, Stein S, Rimes C. Posthospitalization home health care use and changes in functional status in a Medicare population. Med Care 2000;38:494-507.

23. González-Cobos CL, Villalba M, Castaños JG, Pinilla B, Muiño A. Perfil de la asistencia en urgencias entre mayores y menores de 65 años. Rev Esp Geriatr Gerontol 1995;30:355-359.

24. García González G, Cueto-Felgueroso J, García CA, Menéndez AG. La hospitalización a domicilio: una alternativa a la hospitalización convencional. Medicina Integral 1995;25:151155.

25. Naylor MD, Brooten D, Campbell R, Jacobsen BS, Mezey MD, Pauly MV, et al. Comprehensive discharge planning and home follow-up of hospitalized elders: a randomized clinical trial. JAMA 1999;281:613-620.

26. Stessman J, Hammerman-Rozenberg R, Cohen A. Home hospitalization in the spectrum of community geriatric care. Disabil Rehabil 1997;19:134-141.

27. Burton L C, Leff B, Harper M, Ghoshtagore I, Steinwachs DA, Greenough WB 3rd, et al. Acceptability to patients of a home hospital. J Am Geriatr Soc 1998;46:605-609.

28. Minardi Mitre CR. Morales MSV, Fos SC, Llopis AG, Dias JA, Ramon E. La atención domiciliaria como eje de la integración y coordinación de la asistencia sanitaria. Eur J Clin Pharm 2001;3:140-147.

29. Vilà A. Las políticas autonómicas para personas mayores. II. AGATHOS: Atención Sociosanitaria y Bienestar 2001;1:15-29.

30. Llordachs F, Kase K, Sullà E. Reflexions sobre les aliances estratègiques en el sistema sanitari. Full Econòmics 1998;32:6-12.

31. Boling PA. The value of targeted case management during transitional care. JAMA 1999; 281:656-657.

32. Gravil JH, Al-Rawas OA, Cotton MM, Flanigan V, Irwin A, Stevenson RD. Home treatment of exacerbations of chronic obstructive pulmonary disease by an acute respiratory assessment service. Lancet 1998;351:18531855.

33. Minardi Mitre Cotta R, Morales Suarez-Varela M, Llopis Gonzalez A, Cotta Filho JS, Dias
Ricos JA, Real ER. La hospitalización domiciliaria: antecedentes, situación actual y perspectiva. Rev Panam Salud Publica 2001;10: 45-55.

34. LoFaso V. The doctor-patient relationship in the home. Clin Geriatr Med 2000;16:83-94.

35. Litvak J. El envejecimiento de la población: un desafío que va más allá del año 2000. Bol Oficina Sanit Panam 1999;109:1-5.

36. Steiner A, Walsh B, Pickering RM, Wiles R, Ward J, Brooking JI. Therapeutic nursing or unblocking beds? A randomised controlled trial of a post-acute intermediate care unit. [Commentary: Problems with randomised consent. Authors'reply]. BMJ 2001;322:460.

37. Estéves PMH, Santos GAR, Brito EN, Rosario MAB, Freire GG. Propuesta de un modelo de organización de atención domiciliaria basada en atención primaria. Cuadernos de Gestión 1999;5:31-42.

38. Criado-Montilla J, Ibañez-Bermúdez F. Relaciones entre hospital y atención primaria. Experiencia de un servicio de medicina interna. Med Clin (Barc) 1996;106:463-468.

39. Tomiak M, Berthelot JM, Mustard CA. A profile of health care utilization of the disabled population in Manitoba. Med Care 1998;36: 1383-1397.

40. Pardo Crespo MR, Pérez Iglesias R, Llorca J, Rodrigo Calabia E, Álvarez Granda L, DelgadoRodríguez M. Papel de los equipos de atención primaria en la hospitalización infantil de los niños menores de 2 años. Atención Primaria 2000;26;464-467.

41. Mediano C, Guillén M, Aranda E, Pérez F, Peiró S. Como en casa en ningún sitio. Satisfacción de los pacientes hospitalizados a domicilio. Rev Calidad Asistencial 1995;1: 13-18.

42. Programa de atención a las personas mayores. INSALUD, Ministerio de Sanidad y Consumo, Subdirección General de Coordinación Administrativa. Madrid 1999. [Sitio en Internet]. Disponible en: http://www.msc.es/ insalud/(Acceso el 10 de abril de 2002).

43. Shepperd S, Iliffe S. Effectiveness of hospital at home compared to in-patient hospital care. En: Bero L, Grilli R, Grimshaw J, Osman A, eds. Collaboration on effective professional practice module of the Cochrane Database of Systematic Reviews. The Cochrane Collaboration Issue 1. Oxford: Updated Sofware; 1998.

44. Coleman BJ. European models of long-term care in the home and community. Int J Health Serv 1995;25:455-474.
45. González MDD, coord. Hospitalización a domicilio. España: Hoechst Marion Roussel; s/f.

46. Jové N, Bugés J, Dorado MJ, Fernández MA, Rodríguez E. Hospitalización a domicilio. Jano 1999;57:35-38.

47. Iliffe S. Hospital at home: from red to amber? Data that will reassure advocates but without satisfying the sceptics. BMJ 1998;316:17611762.

48. Van der Linder BA. Transmural care: facts and future. En: Schrijvers AJP, ed. Health and health care in the Netherlands. Utrecht: De Tijdstroom; 1997.

49. Pritchard P, Huges J. Shared care. A future imperative? London: Royal Society of Medicine Press, 1995.

50. Ribas E, Protela E. Optimizar los recursos y la gestión de los servicios sanitarios. En: Informe SESPAS. 2001 Nov 22-24; Zaragoza, España; 2002. pp. 357-361.

51. Organización para la Cooperación y el Desarrollo Económico. Health Data File (2001).

52. Gonzáles BLV, Urbanos RMG. Prioridades en la organización de la atención a la salud en España. Informes SESPAS 2002. 2001 Nov 22-24; Zaragoza, España; 2002. pp.1-25.

53. Matezans R. Integración entre niveles asistenciales. Jano 1999;56:12.

54. Bernabei R, Landi F, Gambassi G, Sgadari A, Zuccala G, Mor V, et al. Randomized trial of impact of model of integrated care and case management for older people living in the community. BMJ 1998;316:1348-1351.

55. Landi F, Gambassi G, Pola R, Tabaccanti S, Cavinato T, Carbonin PU, et al. Impact of integrated home care services on hospital use. J Am Geriatr Soc 1999;47:1430-1434.

56. Casado D, López G. Vejez, dependencia y cuidados de larga duración. Situación actual y perspectivas de futuro. Colección Estudios Sociales, No. 6. Barcelona: Fundació "La Caixa"; 2001.

57. Baztán JJ, Gil L, Andrés E. Actividad comunitaria de un servicio de geriatría hospitalario: un ejemplo práctico de coordinación entre atención primaria y especializada. Atención Primaria 2000;26:374-382.

Manuscrito recibido el 9 de noviembre de 2001. Aceptado para publicación, tras revisión, el 4 de marzo de 2002. 
ABSTRACT Objective. Today, the most important demographic change taking place is the rapid aging of the population. While this phenomenon is having a growing and profound impact on all spheres of society, its greatest impact is in the health area, affecting all

\section{Home hospitalization in light of demographic changes and new health challenges}

levels of health care and leading to the need for new resources and new structures. Out of that, in many countries have come different alternatives as well as in-patient, outpatient, and in-home programs that are geared toward improving health care and helping set priorities. One of these new initiatives is home hospitalization, or home health care. One objective of this study was to describe and analyze the characteristics of a population in Spain that was assisted through home hospitalization $(\mathrm{HH})$. Another objective of the study was to comment on the role that $\mathrm{HH}$ can play as a mechanism for integration and coordination between health care levels, in the face of the challenges occurring with the reorganization of health care policies and programs, especially those directed at the elderly.

Methods. A retrospective descriptive study was done of patients assisted through $\mathrm{HH}$ in Sanitary Area 9 of the Autonomous Community of Valencia, which is on the eastern coast of Spain. The area's population was 321 361, of whom 60079 (18.7\%) were 60 or older, including 43044 (13.4\%) who were 65 or older. A descriptive study of the analyzed variables was done, with the mean and standard deviation being computed for quantitative variables, and the absolute and relative frequencies (percentages) being calculated for the qualitative variables.

Results. Of the patients studied, $78 \%$ of them were 65 or older, with an average age of 73 years. They were predominantly women. Of the total group, $72 \%$ of them had chronic diseases, and $67 \%$ had at least one associated secondary diagnosis. There was an important problem of communication between the two principal levels of care, primary care and hospital care, which obviously had an impact on the patients and on the quality and effectiveness of their health care. Furthermore, it was found that $\mathrm{HH}$ finds its greatest utility with and is an effective tool for an adult or elderly population that has multiple chronic degenerative or terminal diseases.

Conclusions. The results of this study highlight the need to create or strengthen channels and mechanisms for interinstitutional communication that will guarantee continuity of care. The ongoing, effective care of the health and well-being of elderly persons requires different levels of health interventions. This care should be comprehensive, adequate, integrated, of high quality, humanized, timely, and coordinated between the two principal levels of health care. In the final analysis, these factors will determine the quality of the health care for geriatric patients and the capacity to solve their health problems. 